\title{
Ethanol Enhances Neurosteroidogenesis in Hippocampal Pyramidal Neurons by Paradoxical NMDA Receptor Activation
}

\author{
Kazuhiro Tokuda, ${ }^{1}$ Yukitoshi Izumi, ${ }^{1}$ and Charles F. Zorumski ${ }^{1,2}$ \\ Departments of ${ }^{1}$ Psychiatry and ${ }^{2}$ Neurobiology, Washington University School of Medicine, St. Louis, Missouri 63110
}

Using an antibody against $5 \alpha$-reduced neurosteroids, predominantly allopregnanolone, we found that immunostaining in the CA1 region of rat hippocampal slices was confined to pyramidal neurons. This neurosteroid staining was increased following $15 \mathrm{~min}$ administration of $60 \mathrm{~mm}$ but not $20 \mathrm{~mm}$ ethanol, and the enhancement was blocked by finasteride and dutasteride, selective inhibitors of $5 \alpha$-reductase, a key enzyme required for allopregnanolone synthesis. Consistent with a prior report indicating that $N$-methyl-D-aspartate (NMDA) receptor (NMDAR) activation can promote steroid production, we observed that D-2-amino-5-phosphonovalerate (APV), a competitive NMDAR antagonist, blocked the effects of $60 \mathrm{~mm}$ ethanol on staining. We previously reported that $60 \mathrm{~mm}$ ethanol inhibits the induction of long-term potentiation (LTP), a cellular model for memory formation, in the CA1 region. In the present study, LTP inhibition by $60 \mathrm{~mm}$ ethanol was also overcome by both the $5 \alpha$-reductase inhibitors and by APV. Furthermore, the effects of ethanol on neurosteroid production and LTP were mimicked by a low concentration of NMDA $(1 \mu \mathrm{M})$, and the ability of NMDA to inhibit LTP and to enhance neurosteroid staining was reversed by finasteride and dutasteride, as well as by APV. These results indicate that ethanol paradoxically enhances GABAergic neurosteroid production by activation of unblocked NMDARs and that acute LTP inhibition by ethanol represents a form of NMDAR-mediated metaplasticity.

\section{Introduction}

During acute ethanol intoxication, individuals can perform complex acts for which they have no recollection, a state referred to as a "blackout" (White, 2003). Understanding how this dense amnesia occurs is important for understanding alcoholism as a neurocognitive disorder. Ethanol is thought to impair memory by inhibiting long-term potentiation (LTP), a form of synaptic plasticity associated with memory processing (Bliss and Collingridge, 1993; Martin et al., 2000). Although ethanol is a partial N-methyl-D-aspartate (NMDA) receptor (NMDAR) antagonist (Izumi et al., 2005), ethanol's effects on LTP involve enhanced GABA-mediated inhibition via $5 \alpha$-reduced neurosteroids, including $3 \alpha$-hydroxy- $5 \alpha$-prenan20-one (allopregnanolone) (Izumi et al., 2007). It is presently unclear which neural cells are responsible for neurosteroidogenesis and how ethanol promotes steroid production.

In this study, we pursued the hypothesis that ethanol's effects on LTP represent a form of metaplasticity involving unblocked NMDARs (Izumi et al., 1992a,b). We based these studies on the findings that ethanol alters extracellular glutamate levels (Moghaddam and Bolinao, 1994; Chefer et al., 2011) and is a partial

Received March 31, 2011; revised May 6, 2011; accepted May 24, 2011.

Author contributions: Y.I. designed research; K.T. and Y.I. performed research; K.T. and Y.I. analyzed data; K.T., Y.I., and C.Z. wrote the paper.

This work was supported by NIH Grants MH07791, GM47969, and AA017413 and the Bantly Foundation. The authors declare no competing financial interests.

Correspondence should be addressed to Yukitoshi Izumi, Department of Psychiatry, Washington University School of Medicine, 660 South Euclid Avenue, St. Louis, M0 63110. E-mail: izumiy@wustl.edu.

DOI:10.1523/JNEUROSCI.1660-11.2011

Copyright $\odot 2011$ the authors $\quad 0270-6474 / 11 / 319905-05 \$ 15.00 / 0$
NMDAR antagonist in the hippocampus (Izumi et al., 2005). We found that both the enhancement of neurosteroid levels and inhibition of LTP by ethanol in CA1 hippocampal pyramidal neurons involve NMDAR activation, not inhibition, and that a low micromolar concentration of NMDA mimics the effects of ethanol on neurosteroidogenesis and LTP.

\section{Materials and Methods}

Animals. Protocols for animal use were approved by the Washington University Animal Studies Committee in accordance with the NIH guidelines for care and use of laboratory animals.

Hippocampal slice preparation. Hippocampal slices were prepared as descried previously (Zorumski et al., 1996) from postnatal day 30-32 male Sprague Dawley rats purchased from Harlan. Rats were anesthetized with isoflurane and decapitated. Slices were cut transversely into $500 \mu \mathrm{m}$ slices using a rotary slicer in artificial CSF (ACSF) containing the following (in mM): $124 \mathrm{NaCl}, 5 \mathrm{KCl}, 2 \mathrm{MgSO}_{4}, 2 \mathrm{CaCl}_{2}, 1.25 \mathrm{NaH}_{2} \mathrm{PO}_{4}, 22$ $\mathrm{NaHCO}_{3}$, and 10 glucose, bubbled with $95 \% \mathrm{O}_{2} / 5 \% \mathrm{CO}_{2}$ at $4-6^{\circ} \mathrm{C}$. Acutely prepared slices were placed on nylon mesh in $10 \mathrm{ml}$ beakers containing gassed ACSF and maintained for at least $1 \mathrm{~h}$ at $30^{\circ} \mathrm{C}$ before experiments.

Immunohistochemistry. Hippocampal slices used for immunohistochemistry were initially screened by electrophysiology to diminish slice-to-slice variability in staining for $5 \alpha$-reduced neurosteroids. Immunostaining was performed as described previously (Tokuda et al., 2010). Slices were incubated with various reagents in separate $10 \mathrm{ml}$ beakers. For experiments (including electrophysiology) in which we examined the role of $5 \alpha$-reductase in the effects of ethanol or NMDA, inhibitors of the enzyme were administered for at least 15 min before the other agents. This was done to allow time for the enzyme inhibitors to access their intracellular sites of action. Following drug treatment, slices 
were fixed in fresh $4 \%$ paraformaldehyde in PBS for $30 \mathrm{~min}$. Samples were then washed with PBS and incubated in blocking solution ( $1 \%$ donkey serum/PBS) for $2 \mathrm{~h}$ at $25^{\circ} \mathrm{C}$. Slices were incubated with a primary antibody raised in sheep against $5 \alpha$-reduced neurosteroids diluted 1:2500 in blocking solution for $48 \mathrm{~h}$ at $4^{\circ} \mathrm{C}$. The polyclonal antibody against $5 \alpha$ steroids has minimal cross-reactivity with other neurosteroids in rats (Bernardi et al., 1998).

After incubation with primary antibody, slices were rinsed with PBS and incubated with a secondary antibody, Alexa Fluor 488 donkey anti-sheep IgG (diluted 1:500), for $2 \mathrm{~h}$ at $25^{\circ} \mathrm{C}$. After staining, slices were washed with PBS and mounted onto microscope slides with Fluoromount-G (Southern Biotech).

Confocal images were obtained using a $60 \times$ objective (1.4 numerical aperture), a C1 laser scanning confocal microscope, and Z-C1 software (Nikon Instruments). All parameters were kept constant within an experiment. Digital images were analyzed, and the average intensity of the tissue was measured using MetaMorph software (Universal Imaging).

Extracellular field potential recording. For electrophysiology, slices were incubated in a submerged recording chamber with continuous bath perfusion of oxygenated ACSF at 2 $\mathrm{ml} / \mathrm{min}$ at $30^{\circ} \mathrm{C}$. Extracellular recordings were obtained from the apical dendritic layer of the CA1 region elicited with $0.1 \mathrm{~ms}$ constant current pulses through a bipolar stimulating electrode (Rhodes Medical Instruments) in stratum radiatum. LTP was induced by applying a single 100 $\mathrm{Hz} \times 1$ s high-frequency stimulation (HFS) using a $50 \%$ maximal stimulus. Input-output curves were repeated 60 min following HFS for statistical comparisons of changes in EPSP slopes at the half-maximal point. Signals were digitized and analyzed using PCLAMP software (Molecular Devices).

Statistical analysis. All data are expressed as mean \pm SEM. Student's $t$ test was used for comparisons between two groups. If an equal variance test failed, the nonparametric Mann-Whitney rank sum test was applied. For multiple comparisons, ANOVA followed by post hoc Holm-Sidak test was used. Statistical analyses were performed using commercial software (Sigma Stat 3.11; Systat Software). $p$ values of $<0.05$ were considered statistically significant.

Materials. Finasteride was obtained from Steraloids. Dutasteride was obtained from AK Scientific. All other chemicals were purchased from Sigma. Anti-allopregnanolone antisera were purchased from Dr. Robert Purdy, University of California-San Diego, La Jolla, CA. Alexa Fluor 488 was purchased from Invitrogen.

\section{Results}

We initially examined the effects of ethanol on neurosteroid immunostaining in the CA1 region of rat hippocampal slices by using an antibody against $5 \alpha$-reduced steroids, predominantly allopregnanolone. Consistent with earlier reports (Saalman et al., 2007; Tokuda et al., 2010), we found that steroid staining was largely, if not exclusively, confined to CA1 pyramidal neurons. Ethanol increased neurosteroid staining in a concentrationdependent fashion, with significant effects following $15 \mathrm{~min}$ administration of $60 \mathrm{~mm}\left(244.7 \pm 39.5 \%\right.$ vs control, $n=5,{ }^{\star} p<$ 0.001 ) (Fig. $1 A, B$ ) but not $20 \mathrm{~mm}$ ethanol (108.6 $\pm 7.0 \%$ ) (data not shown). The enhanced staining was blocked completely by 1 $\mu \mathrm{M}$ finasteride and $1 \mu \mathrm{M}$ dutasteride, selective inhibitors of $5 \alpha$ reductase, a key enzyme required for neurosteroid synthesis

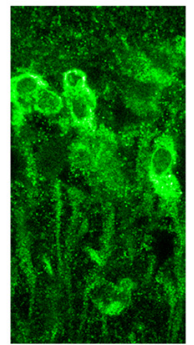

$60 \mathrm{mM}$ Ethanol

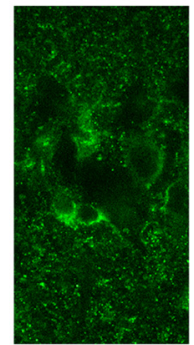

Ethanol + Finasteride

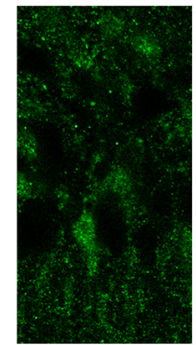

Ethanol + Dutasteride

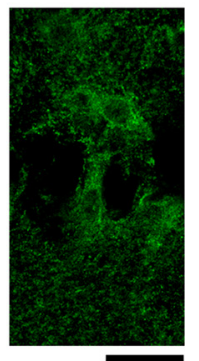

Ethanol
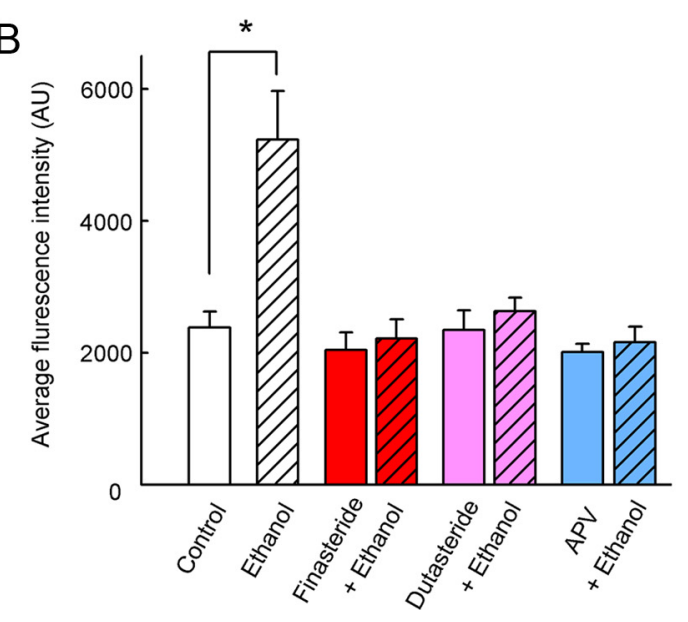

Figure 1. Ethanol-mediated neurosteroidogenesis results from activation of NMDARs and $5 \alpha$-reductase. $\boldsymbol{A}$, Immunostaining against $5 \alpha$-reduced neurosteroids was observed in cell bodies of pyramidal neurons in naive hippocampal slices. The neurosteroid staining was enhanced by 15 min incubation with $60 \mathrm{~mm}$ ethanol but blocked by $1 \mu \mathrm{m}$ finasteride, $1 \mu \mathrm{m}$ dutasteride, and $50 \mu \mathrm{m}$ APV. Scale bar, $25 \mu \mathrm{m}$. $B$, Summary of immunostaining studies shows fluorescence intensity (arbitrary units) as mean \pm SEM. $p$ values are calculated by Holm-Sidek post hoc test, $n=5$; ${ }^{*} p<0.001$.

(97.4 $\pm 16.1 \%$ and $107.5 \pm 14.7 \%, n=5$, respectively) (Fig. $1 A, B)$ (Aggarwal et al., 2010).

We subsequently sought to understand how ethanol enhances neurosteroidogenesis. Based on reports indicating that NMDAR activation promotes steroid production in the hippocampus (Kimoto et al., 2001) and that ethanol promotes release of glutamate (Chefer et al., 2011), we examined the effects of NMDAR inhibition on neurosteroid staining. Administration of $50 \mu \mathrm{M}$ D-2-amino-5-phosphonovalerate (APV), a broad spectrum competitive NMDAR antagonist, did not alter baseline staining $(93.0 \pm 11.4 \%$ vs control, $n=5)$ (Fig. $1 B$ ) but completely prevented the effects of $60 \mathrm{~mm}$ ethanol $(88.9 \pm 12.9 \%, p=0.008$ vs $60 \mathrm{~mm}$ ethanol alone) (Fig. $1 A, B)$.

We also found that $60 \mathrm{~mm}$ but not $20 \mathrm{~mm}$ ethanol inhibited LTP induction (Izumi et al., 2005, 2007), and this LTP inhibition was overcome by $1 \mu \mathrm{M}$ finasteride (EPSP change: $139.2 \pm 3.4 \%$ of baseline, $n=5$ ) (Fig. $2 A$ ) and $1 \mu \mathrm{M}$ dutasteride (EPSP change: $154.0 \pm 8.1 \%$ of baseline, $n=5$ ) (Fig. $2 A$ ). Exploiting an earlier observation that ethanol's block of LTP persists for over an hour after drug washout (Izumi et al., 2005), we also found that effects on LTP were reversed by coadministration of $50 \mu \mathrm{M}$ APV (EPSP change: $149.5 \pm 7.6 \%$ of baseline, $n=5$ ) (Fig. $2 B$ ). Administration of APV alone $30 \mathrm{~min}$ before HFS followed by washout had no effect on LTP induction (EPSP change: $157.7 \pm 9.2 \%$ of baseline, $n=5$, data not shown).

Because these results indicate that ethanol's effects on neurosteroid production and LTP involve activation of NMDARs, we 


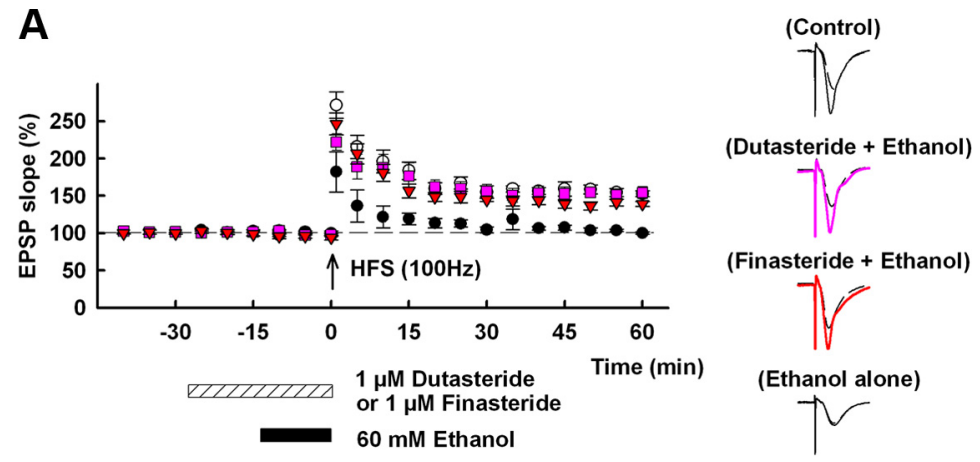

B

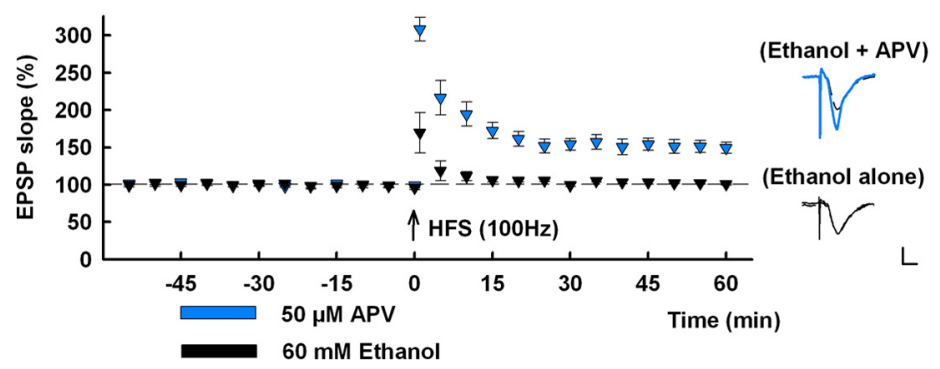

Figure 2. Ethanol-mediated LTP inhibition involves activation of NMDARs and $5 \alpha$-reductase. $\boldsymbol{A}$, In control slices, LTP is readily induced (white circles) by a $100 \mathrm{~Hz} \times 1$ shigh-frequency stimulation (HFS, arrow). Fifteen minute administration of $60 \mathrm{~mm}$ ethanol (black bar) inhibited LTP induction (black circles), while finasteride (red triangles) or dutasteride (pink squares) overcame LTP inhibition. $\boldsymbol{B}$, Ethanol's inhibition of LTP (black triangles) persisted for at least $30 \mathrm{~min}$ after washout (black bar). This inhibitory effect was prevented by coadministration of APV (blue bar) with ethanol (blue triangles). Traces depict EPSPs before (dashed lines) and $60 \mathrm{~min}$ after HFS (solid lines). Calibration, $1 \mathrm{mV}, 5 \mathrm{~ms}$.

also examined the effects of a low concentration of NMDA (Izumi et al., 1992a,b). When administered at $1 \mu \mathrm{M}, \mathrm{NMDA}$, like ethanol, enhanced neurosteroid staining in pyramidal neurons (244.2 $\pm 22.8 \%$ vs control, $n=5,{ }^{\star} p<0.001$ ) (Fig. $\left.3 A, B\right)$. Also similar to ethanol, the enhancement of neurosteroid staining by NMDA was reversed by finasteride and dutasteride (102.3 \pm $8.0 \%$ and $110.6 \pm 6.6 \%, n=5$, respectively) (Fig. $3 A, B$ ). Furthermore, $1 \mu \mathrm{M}$ NMDA administered before tetanic stimulation inhibited the induction of LTP (EPSP change: $100.2 \pm 8.5 \%$ of baseline, $n=5$ ) (Fig. $4 A$ ), and this effect was also overcome by finasteride and dutasteride (EPSP change: $157.6 \pm 10.9 \%$ of baseline, $153.4 \pm 5.8 \%$ of baseline, $n=5$, respectively) (Fig. $4 A$ ). Finally, NMDA also had persistent effects on LTP, lasting at least 30 min following washout (EPSP change: $101.3 \pm 2.8 \%$ of baseline, $n=5$ ) (Fig. $4 B$ ). Again, similar to ethanol, APV coadministration with NMDA blocked the LTP inhibition (EPSP change: $157.7 \pm 9.2 \%$ of baseline, $n=5$ ) (Fig. $4 B$ ).

\section{Discussion}

Ethanol has previously been shown to increase neurosteroid levels in the isolated hippocampus (Sanna et al., 2004), although it has remained unclear which cells are responsible for steroid production and how this effect occurs. Pyramidal neurons in the CA1 region express the machinery for cholesterol trafficking and neurosteroid synthesis (Kimoto et al., 2001; Agís-Balboa et al., 2006), and these neurons are the principal if not exclusive cells in the region that are immunopositive for allopregnanolone and other $5 \alpha$-reduced neurosteroids under physiological conditions (Saalman et al., 2007; Tokuda et al., 2010). We found that CA1 pyramidal neurons are also the cells that show enhanced neurosteroid staining following exposure to concentrations of ethanol that impair LTP. Interestingly, the acute effects of ethanol on neurosteroidogenesis, like effects on LTP, require high concentrations with no effect at $20 \mathrm{~mm}$ but significant effects at $60 \mathrm{~mm}$. Thus, if neurosteroids are involved in the negative effects of ethanol on cognitive function, it is likely that only high concentrations of ethanol acutely produce these effects because of the apparent threshold for neurosteroid synthesis. Based on the observation that both enhanced steroid production and LTP inhibition are prevented by $5-\alpha$ reductase inhibitors (Izumi et al., 2007), our results provide further support for the hypothesis that high concentrations of ethanol impair hippocampal LTP and presumably cognitive functions through neurosteroidogenesis.

The present studies also provide insights into how ethanol alters neurosteroid levels and LTP. While it is widely recognized that ethanol antagonizes NMDARs acutely, its effects are only partial even at concentrations of 50-100 mM (Lovinger et al., 1989). We previously found that at concentrations up to $60 \mathrm{~mm}$ ethanol partially depresses NMDAR-mediated EPSPs in the CA1 region. These effects are mimicked and occluded by ifenprodil, a selective NR1/NR2B antagonist (Izumi et al., 2005), suggesting that ethanol preferentially antagonizes a subtype of NMDARs in this region. Interestingly, CA1 LTP is not blocked by ifenprodil (Liu et al., 2004, Izumi et al., 2006), and partial ( 50\%) inhibition of NMDAR EPSPs does not depress LTP induction (Izumi et al., 2006). Thus, it is unlikely that ethanol blocks LTP induction solely through effects on NMDARs. Consistent with this, ethanol's block of LTP is prevented by inhibition of $\mathrm{GABA}_{\mathrm{A}}$ receptors (Izumi et al., 2005).

We previously found that administration of low concentrations of NMDA before tetanic stimulation dampens LTP induction via a metaplastic effect (Izumi et al., 1992a,b), indicating that low-level but sustained NMDAR activation paradoxically inhibits an NMDAR-dependent form of synaptic plasticity. Based on studies showing that ethanol can alter extracellular glutamate (Moghaddam and Bolinao, 1994; Chefer et al., 2011), we postulated that ethanol may produce its effects on LTP by a similar metaplastic mechanism. How ethanol influences extracellular glutamate levels remains uncertain. Based on our prior work, it does not appear that ethanol acutely increases glutamate release at CA1 synapses, because ethanol does not alter EPSP pairedpulse plasticity in hippocampal slices (Murayama et al., 2006). It is possible that ethanol alters extracellular glutamate via release from glia, as has been shown in hippocampal astrocytes (Salazar et al., 2008), or by effects on glutamate uptake (Othman et al., 2002, Melendez et al., 2005).

The present results support the idea that ethanol's effects on both neurosteroidogenesis and LTP result from activation of unblocked NMDARs. Indeed, we found that both effects of ethanol were prevented by complete NMDAR inhibition. Moreover, we observed that brief administration of $1 \mu \mathrm{M}$ NMDA in the presence of extracellular magnesium is sufficient to facilitate neurosteroidogenesis in CA1 pyramidal neurons and block LTP. While we did not examine the concentration-response for NMDA's 
effects on steroid immunostaining, we found that low-level NMDAR activation is sufficient to increase neurosteroid staining in pyramidal neurons. Both effects of NMDA are also reversed by NMDAR antagonism (Izumi et al., 1992a,b) and $5 \alpha$-reductase inhibitors, providing strong parallels to the effects of ethanol. These results further suggest that low-level but persistent NMDAR activation is an important regulator of local neurosteroid production and synaptic plasticity, acting through an as yet unknown signaling pathway that could include calcineurin, nitric oxide synthase, and p38 MAP kinase, molecules previously linked to NMDAR-mediated LTP inhibition (Izumi et al., 2008). We note, however, that a recent study found that ethanol facilitates neurosteroid production in vivo through release of adrenocorticotropic hormone and synthesis of steroidogenic acute regulatory protein (Boyd et al., 2010). Thus, mechanisms other than direct regional synthesis via NMDAR activation contribute to ethanol's overall effects on neurosteroid generation. Furthermore, ethanol alters release of other signaling molecules, including GABA, and these agents could also contribute to modulation of LTP (Siggins et al., 2005). Additionally, the effects of ethanol on LTP take longer than NMDA $(\sim 15 \mathrm{~min}$ compared to $5 \mathrm{~min}$ ), and thus there are some differences between these agents in their metaplastic actions.

We conclude that ethanol, despite being a partial NMDAR antagonist (Izumi et al., 2005), inhibits LTP induction via enhancement of neurosteroidogenesis triggered by activation of unblocked NMDARs in CA1 hippocampal pyramidal neurons. Thus, acute ethanol-induced LTP inhibition appears to represent a form of NMDAR-mediated metaplasticity (Izumi et al., 1992a,b). These results suggest novel strategies to prevent the cognitive impairment resulting from acute ethanol intoxication and perhaps to prevent the longer-term cognitive consequences of alcoholism.

\section{References}

Aggarwal S, Thareja S, Verma A, Bhardwaj TR, Kumar M (2010) An overview on 5alpha-reductase inhibitors. Steroids 75:109-153.

Agís-Balboa RC, Pinna G, Zhubi A, Maloku E, Veldic M, Costa E, Guidotti A (2006) Characterization of brain neurons that express enzymes mediating neurosteroid biosynthesis. Proc Natl Acad Sci USA 103:14602-14607.

Bernardi F, Salvestroni C, Casarosa E, Nappi RE, Lanzone A, Luisi S, Purdy RH, Petraglia F, Genazzani AR (1998) Aging is associated with changes in allopregnanolone concentrations in brain, endocrine glands and serum in male rats. Eur J Endocrinol 138:316-321.

Bliss TV, Collingridge GL (1993) A synaptic model of memory: long-term potentiation in the hippocampus. Nature 361:31-39.

Boyd KN, Kumar S, O'Buckley TK, Porcu P, Morrow AL (2010) Ethanol induction of steroidogenesis in rat adrenal and brain is dependent upon pituitary ACTH release and de novo adrenal StAR synthesis. J Neurochem 112:784-796.

Chefer V, Meis J, Wang G, Kuzmin A, Bakalkin G, Shippenberg T (2011) Repeated exposure to moderate doses of ethanol augments hippocam- pal glutamate neurotransmission by increasing release. Addict Biol $16: 229-237$.

Izumi Y, Clifford DB, Zorumski CF (1992a) Low concentrations of $N$-methyl-D-aspartate inhibit the induction of long-term potentiation in rat hippocampal slices. Neurosci Lett 137:245-248.

Izumi Y, Clifford DB, Zorumski CF (1992b) Inhibition of long-term potentiation by NMDA-mediated nitric oxide release. Science 257:1273-1276.

Izumi Y, Nagashima K, Murayama K, Zorumski CF (2005) Acute effects of ethanol on hippocampal long-term potentiation and long-term depression are mediated by different mechanisms. Neuroscience 136:509-517.

Izumi Y, Auberson YP, Zorumski CF (2006) Zinc modulates bidirectional hippocampal plasticity by effects on NMDA receptors. J Neurosci 26:7181-7188.

Izumi Y, Murayama K, Tokuda K, Krishnan K, Covey DF, Zorumski CF (2007) GABAergic neurosteroids mediate the effects of ethanol on long-term potentiation in rat hippocampal slices. Eur J Neurosci 26:1881-1888.

Izumi Y, Tokuda K, Zorumski CF (2008) Long-term potentiation inhibition by low level $N$-methyl-D-aspartate receptor activation involves calcineurin, nitric oxide and p38 MAP kinase. Hippocampus 18:258-265.

Kimoto T, Tsurugizawa T, Ohta Y, Makino J, Tamura Ho, Hojo Y, Takata N, Kawato S (2001) Neurosteroid synthesis by cytochrome p450-containing systems localized in the rat brain hippocampal neurons: $N$-methyl-Daspartate and calcium-dependent synthesis. Endocrinology 142:3578-3589.

Liu L, Wong TP, Pozza MF, Lingenhoehl K, Wang Y, Sheng M, Auberson YP, Wang YT (2004) Role of NMDA receptor subtypes in governing the direction of hippocampal synaptic plasticity. Science 304:1021-1024.

Lovinger DM, White G, Weight FF (1989) Ethanol inhibits NMDAactivated ion current in hippocampal neurons. Science 243:1721-1724.

Martin SJ, Grimwood PD, Morris RG (2000) Synaptic plasticity and memory: an evaluation of the hypothesis. Annu Rev Neurosci 23:649-711. 
A

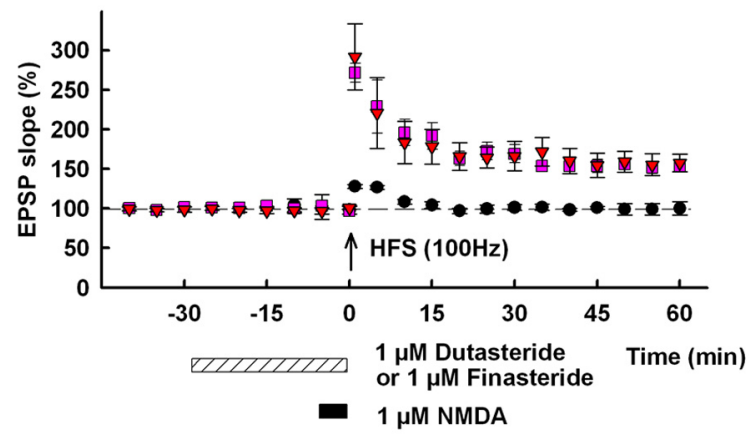

(Dutasteride + NMDA)



B



Figure 4. NMDA-mediated LTP inhibition involves neurosteroidogenesis. $\boldsymbol{A}$, Five minute treatment with $1 \mu \mathrm{M}$ NMDA (black bar) before a high-frequency stimulation (HFS, arrow) was sufficient to block CA1 LTP (black triangles). This LTP inhibition was overcome by $1 \mu \mathrm{m}$ finasteride (red triangles) or $1 \mu \mathrm{m}$ dutasteride (pink squares). $\boldsymbol{B}$, The effects of $1 \mu \mathrm{m}$ NMDA on LTP inhibition lasted at least $30 \mathrm{~min}$ following washout (black triangles). Coadministration of APV (blue bar) overcame NMDA's inhibition of LTP (blue triangle). Traces depict EPSPs before (dashed lines) and 60 min after HFS (solid lines). Calibration, $1 \mathrm{mV}, 5 \mathrm{~ms}$.

Melendez RI, Hicks MP, Cagle SS, Kalivas PW (2005) Ethanol exposure decreases glutamate uptake in the nucleus accumbens. Alcohol Clin Exp Res 29:326-333.

Moghaddam B, Bolinao ML (1994) Biphasic effect of ethanol on extracellu- lar accumulation of glutamate in the hippocampus and nucleus accumbens. Neurosci Lett 178:99-102.

Murayama K, Zorumski CF, Izumi Y (2006) Effects of neurosteroid $3 \alpha$-hydroxy- $5 \alpha$-pregnan-20-one on ethanol-mediated paired-pulse depression of population spikes in the CAl region of rat hippocampal slices. Neurosci Lett 394:28-32.

Othman T, Sinclair CJ, Haughey N, Geiger JD, Parkinson FE (2002) Ethanol alters glutamate but not adenosine uptake in rat astrocytes: evidence for protein kinase C involvement. Neurochem Res 27:289-296.

Saalmann YB, Kirkcaldie MT, Waldron S, Calford MB (2007) Cellular distribution of the $\mathrm{GABA}_{\mathrm{A}}$ receptor-modulating $3 \alpha$-hydrox, $5 \alpha$-reduced pregnane steroids in the adult rat brain. J Neuroendocrinol 19:272-284.

Salazar M, Pariente JA, Salido GM, González A (2008) Ethanol induces glutamate secretion by $\mathrm{Ca}^{2+}$ mobilization and $\mathrm{ROS}$ generation in rat hippocampal astrocytes. Neurochem Int 52:1061-1067.

Sanna E, Talani G, Busonero F, Pisu MG, Purdy RH, Serra M, Biggio G (2004) Brain steroidogenesis mediates ethanol modulation of $\mathrm{GABA}_{\mathrm{A}}$ receptor activity in rat hippocampus. J Neurosci 24:6521-6530.

Siggins GR, Roberto M, Nie Z (2005) The tipsy terminal: presynaptic effects of ethanol. Pharmacol Ther 107:80-98.

Tokuda K, O'Dell KA, Izumi Y, Zorumski CF (2010) Midazolam inhibits hippocampal longterm potentiation and learning through dual central and peripheral benzodiazepine receptor activation and neurosteroidogenesis. J Neurosci 30:16788-16795.

White AM (2003) What happened? Alcohol, memory blackouts and the brain. Alcohol Res Health 27:186-196.

Zorumski CF, Mennerick S, Izumi Y (1996) Assessment of synaptic effects of nitric oxide in hippocampal neurons. Methods Neurosci 31:282-299. 